\title{
Apport d'un modèle murin de réaction du greffon contre l'hôte pour l'étude du lichen plan idiopathique : rôle du ligand de CD95 dans l'induction des lésions buccales
}

\author{
Contribution of a graft versus host disease murine model to the oral lichen \\ planus characterization: role of CD95 ligand for induction of oral lesions
}

CHRISTOPHE DESCHAUMES*/*****, MARTINE BAUDET-POMMEL***, JEAN-CLAUDE AMEISEN*, ANNE JANIN**

\begin{abstract}
RÉSUMÉ
La lésion histologique élémentaire du lichen plan buccal et de la maladie du greffon contre l'hôte (GvHD pour graft versus host disease) après greffe de moelle osseuse résulte d'une apoptose isolée des cellules cibles, associée à un infiltrat effecteur. Ces lésions ont été reproduites, dans un modèle murin, par greffe transfusionnelle de splénocytes ou de lymphocytes T purifiés, allogéniques, chez un receveur immunodéficient. Dans ce modèle, les signes cliniques et pathologiques de la GvHD sont observés de façon reproductible. II a ainsi permis de réaliser une étude histologique séquentielle des lésions lichénoïdes buccales.

L'apoptose, médiée par le ligand de CD95 (CD95L), est incriminée dans l'induction des lésions de GvHD, notamment cutanées. L'absence de lésions apoptotiques après transfusion de splénocytes allogéniques déficients en CD95L, a permis de montrer que les lésions élémentaires apoptotiques des cellules cibles épithéliales, dans les lésions du lichen plan buccal, sont également médiées par l'engagement du ligand de CD95 exprimé par l'infiltrat de lymphocytes T cytotoxiques présents autour des cellules cibles. (Med Buccale Chir Buccale 2003; $9: 149-58)$
\end{abstract}

mots clés : Lichen plan, maladie du greffon contre l'hôte, apoptose

\section{SUMMARY}

Oral lichen planus and oral graft versus host disease (GvHD) after bone marrow transplanatation present identical elementary pathological lesions, i.e. an apoptosis of the basal epithelial cell associated with an infiltrate of mononuclear cells.

In this study, these lesions are reproduced in a murine model which use intra-peritoneally transfers of allogeneic splenocytes or allogeneic purified Tlymphocytes into immunodeficient recipients. Sequencial study of the tongue lichen-like lesions shows the progression of the pathological alterations of the epithelium.

Apoptosis mediated by CD95 ligand (CD95L) is proposed as a mechanism of cutaneous GvHD lesions. In this model, the absence of oral lesions after injection of CD95L deficient splenocytes shows that elementary lichen-like lesions of oral epithelium are mediated by CD95L engagement. CD95L should be expressed by cytotoxic Tlymphocytes infiltrated near the target basal and para-basal cells of oral epithelium. (Med Buccale Chir Buccale 2003; 9:149-58)

key words : lichen planus, graft versus host disease, apoptosis

\footnotetext{
* Laboratoire UA INSERM 9922 - Hôpital Bichât-Claude Bernard - Paris

** Laboratoire d'Anatomie Pathologique - Hôpital Saint Louis - Paris

*** Service d'Odontologie - CHRU Clermont-Ferrand

Demande de tirés à part :

C. Deschaumes Service d'Odontologie Hôtel Dieu CHRU Clermont-Ferrand Bd Léon Malfreyt 63058 Clermont Ferrand Cedex 1
} 
médecine

buccale

chirurgie

buccale

VOL. $9, \mathrm{~N}^{\circ} 3$ 2003

page 150
Le lichen plan représente l'étiologie la plus fréquente des lésions dites "kératosiques » de la muqueuse buccale. Sa prévalence est de 1 à $2 \%$ dans la population générale [1]. L'intérêt de cette pathologie est due à la difficulté de son traitement ainsi qu'à son éventuelle transformation maligne, essentiellement pour les formes érosives et chroniques. Cette transformation est évaluée entre $0.4 \%$ et $1.5 \%$ selon les études [2, 3].

Aux anciennes étiologies - stress, fatigue, agents exogènes irritatifs -, on a substitué une étiologie auto-immune à médiation cellulaire dirigée contre les cellules basales altérées de l'épithélium [4]. La présence accrue de lymphocytes $T$ cytotoxiques [5] et la diminution de la prolifération et de l'activité des lymphocytes $T$ suppresseurs dans les lésions [6] corroborent cette hypothèse. Walsh [7] a montré que les agents exogènes irritatifs peuvent provoquer une altération du phénotype des kératinocytes. Ceux-ci sont alors présentés aux lymphocytes $\mathrm{T}$, par les cellules présentatrices de l'antigène. Les agents exogènes semblent donc favoriser une réaction cytotoxique à médiation cellulaire contre les kératinocytes basaux.

Des lésions lichéniennes buccales [5] sont également observées au cours des phases aiguës et chroniques de la maladie du greffon contre I'hôte [8] (GvHD pour graft versus host disease), complication majeure des greffes de moelle osseuse allogéniques. Ces lésions lichéniennes constituent un des marqueurs les plus constants de la phase chronique de la GvHD et reproduisent, cliniquement et histologiquement, le lichen plan idiopathique [9].

La lésion élémentaire de la GvHD, comparable à celle du lichen plan idiopathique, traduit l'apoptose isolée des cellules épithéliales cibles au contact d'une cellule immune mononucléée allogénique [9]. Les mécanismes cytotoxiques des cellules T, classiquement mis en cause dans l'induction de l'apoptose dans les organes cibles de la GvHD, font intervenir la perforine et la granzyme [10, 11], les recepteurs du TNF [12] et du CD95 ; ce dernier est une protéine membranaire de la famille des récepteurs du TNF $[13,14]$. En se liant avec le récepteur membranaire CD95 des cellules cibles, le ligand de CD95 (CD95L), exprimé par les cellules T cytotoxiques du donneur, déclenche le programme de mort cellulaire de ces cellules cibles. Un travail récent, sur un modèle murin de réaction allogénique mimant la GvHD, a montré que l'engagement du CD95 provoque l'apoptose des cellules endothéliales dans les organes cibles de cette maladie [15].

La ligation de CD95 est également un des mécanismes évoqués dans la pathogénie des lésions épithéliales cutanées du lichen et de la GvHD [16]. Or, des études immunohistochimiques dans le lichen idiopathique ont montré que le récepteur CD95 est sur-exprimé dans les cellules cibles épithéliales des lésions lichéniennes [17]. Aussi, le but de ce travail est d'évaluer dans un modèle murin de réaction allogénique, les mécanismes effecteurs, en particulier le rôle de CD95L, dans la lésion élémentaire buccale qui ressemble à celle du lichen idiopathique.

\section{MATÉRIEL ET MÉTHODES}

\section{Animaux}

Les souris receveuses immunodéficientes SCID/BALB/c $\left(\mathrm{H}-2^{\mathrm{d}}\right)$ ont été fournies par le laboratoire Harlan (Oxon, Angleterre) et celles RAG/C57BL/6 ( $\left.\mathrm{H}-2^{\mathrm{b}}\right)$ par le laboratoire Jackson (Bar Harbor, USA). Elles ont été gardées dans des isolateurs stériles changés toutes les semaines, et elles ont été nourries avec des aliments et de l'eau stériles ad libitum. Les cellules injectées proviennent de souris C57BL/6 $\left(\mathrm{H}-2^{\mathrm{b}}\right)$, issues du laboratoire Iffa Credo (Arbresle, France) et de souris Ipr/C57BL/6 $\left(\mathrm{H}-2^{\mathrm{b}}\right)$ déficientes en CD95 et g/d/C57BL/6 ( $\left.\mathrm{H}-2^{\mathrm{b}}\right)$ issues de laboratoire Jackson. Pour chaque expérience, les souris immunodéficientes mâles, âgées de 7 à 8 semaines, reçoivent par voie intra-péritonéale, dans $500 \mu l$ de milieu RPMI-1640, le nombre souhaité de splénocytes issus de souris d'âge et de sexe identiques.

\section{Validation du modèle de réaction du greffon contre l'hôte}

Une suspension de $2,5.10^{7}$ splénocytes totaux (lymphocytes B, T, cellules NK) allogéniques, issues de souris C57BL/6 a été injectée par voie intrapéritonéale à des souris immunodéficientes 
mâles SCID/BALB/c $\left(H-2^{d}\right)$. Le donneur et le receveur sont histo-incompatibles dans les classes I et II du complexe majeur d'histocompatibilité.

Pour chaque expérience, des contrôles négatifs par injection du même nombre de cellules syngéniques $B A L B / c\left(H-2^{d}\right)$ et par injection de $500 \mu l$ de RPMI-1640 sans cellule ont été simultanément réalisés dans des receveurs congéniques.

Les cellules injectées ont été obtenues après prélèvement et broyage de la rate dans du RPMI1640 stérile, suivi d'une hémolyse dans $\mathrm{NH}_{4} \mathrm{Cl}$. Les monocytes et les macrophages sont déplétés par adhésion après incubation à $37^{\circ} \mathrm{C}$ et avec $5 \%$ de $\mathrm{CO} 2$ pendant 2 heures, dans du RPMI1640 complémenté avec $10 \%$ de sérum de veau fœtal, $2 \mathrm{mM}$ de L-glutamine et $1 \mathrm{mM}$ de pyruvate. Les lymphocytes totaux vivants sont isolés par centrifugation avec gradient de densité (Lympholyte M -TEBU, Santa Cruz, USA). Après lavage, la concentration cellulaire est ajustée pour obtenir le nombre souhaité de cellules dans $500 \mu \mathrm{l}$ de RPMI- 1640 .

Les souris injectées ont été sacrifiées à différents

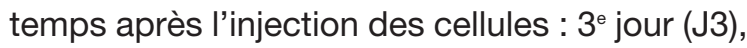
$7^{\mathrm{e}}$ jour (J7), 14 $4^{\mathrm{e}}$ jour (J14) et $28^{\mathrm{e}}$ jour (J28).

\section{Modulation des paramètres de la greffe transfusionnelle Nombre de cellules injectées}

Pour étudier l'influence du nombre de cellules injectées sur la sévérité des lésions, des greffes transfusionnelles, avec $10^{6}, 2,5.10^{7}, 5.10^{7}$ et $10^{8}$. cellules, ont été réalisées sur des cohortes de 4 souris immunodéficientes SCID-BALB/c, en utilisant toujours la même technique de préparation des cellules. Elles ont été sacrifiées à J14, excepté pour les souris ayant reçu $10^{8}$ cellules qui ont été sacrifiées avant leur décès, à J2.

\section{Type de cellules injectées}

Pour vérifier que les lésions obtenues après injection de $2,5.10^{7}$ splénocytes totaux sont dues à l'action cytotoxique des lymphocytes T, l'intensité des lésions a été comparée avec celle obtenue après injection de la fraction équivalente de lymphocytes $T$ purifiés, soit $10^{7}$ cellules. La purification des lymphocytes $T$ a été réalisée après déplétion des lymphocytes $B$ et des cellules NK, avec des billes magnétiques Dynabeads (Dynal, Oslo, Norvège), après incubation des cellules à $4^{\circ} \mathrm{C}$ pendant 30 minutes, avec les anticorps monoclonaux CD19 (1D3) et NK cells (2B4) antisouris fournis par le laboratoire Pharmingen (San Diego, USA).

La quantification des populations cellulaires et le contrôle de la pureté de la suspension cellulaire ont été faites par examen cytofluorométrique avec un FACS Becton Dickinson (San Jose, CA). Les anticorps monoclonaux anti-souris utilisés sont Thy1-2 (53-2.1 ; PE), B220 (RA3-6B2; CyChrome), CD8 (53-6.7 ; FITC), CD4 (RM4-5 ; FITC) et ont également été fournis par le laboratoire Pharmingen. Les souris ont été sacrifiées à J14.

\section{Etude des voies de l'apoptose}

Les souris g/d/C57BL/6 présentent une mutation spontanée induisant l'expression, par les populations lymphocytaires T, de CD95L non fonctionnel [18]. La mutation Ipr du même fond génétique s'accompagne d'une déficience d'expression de la protéine CD95 dans les populations lymphocytaires avec sur-expression de CD95L fonctionnel [19]. Pour évaluer si l'intensité des lésions est fonction du potentiel d'expression de CD95L par les lymphocytes T du donneur, on a transféré $2,5.10^{7}$ splénocytes allogéniques déficients en CD95L ( $g / d / C 57 B L / 6)$ ou en CD95 (Ipr/C57BL/6) chez des receveurs immunodéficients allogéniques SCID-BALB/c.

Des contrôles négatifs ont été réalisés par transfert du même nombre de splénocytes /pr/C57BL/6 ou gld/C57BL/6 dans des receveurs syngéniques SCID-C57BL/6. Les dissections et les analyses pathologiques systématiques ont été réalisées à J14.

\section{Prélèvements et analyse pathologique Examen macroscopique}

Après l'injection des cellules, les souris sont pesées 2 fois par semaine et le degré d'atteinte clinique est apprécié selon les critères retenus par Cooke [20]. Pour cela, le poids, l'activité, la posture, l'état du pelage des souris et l'atteinte macroscopique de leur peau sont évalués et cotés selon une échelle variant de 0 à 2 en fonction de leur altération. Le degré d'atteinte clinique cor- médecine

buccale

chirurgie

buccale

VOL. $9, \mathrm{~N}^{\circ} 3$ 2003

page 151 
médecine

buccale chirurgie buccale

VOL. $9, \mathrm{~N}^{\circ} 3$ 2003

page 152 respond à la somme de ces scores et est donc gradé de 0 à 10 .

Les souris ont été sacrifiées par dislocation cervicale et autopsiées. La muqueuse buccale est prélevée au niveau de la langue et une partie est fixée dans une solution alcoolique de formol pour examen en microscopie photonique. Les prélèvements ainsi fixés sont inclus en paraffine et coupés à $3 \mu \mathrm{m}$ d'épaisseur. Les coupes sont colorées à l'hématine-éosine.

Pour l'observation en microscopie électronique, une partie du prélèvement est fixée dans du glutaraldéhyde à $2,5 \%$, puis rincée dans du cacodylate. Les tissus sont ensuite post-fixés dans du tétroxyde d'osmium puis déshydratés et enrobés dans de la résine Taab Epon 812 (Marivac, Nova Scotia, Canada). Les coupes ultrafines sont colorées avec de l'acétate d'uranyl et du nitrate de bismuth, puis examinées avec un microscope électronique à transmisssion Jeol 100B.

\section{Examen microscopique}

A l'examen en microscopie optique, les signes histologiques de GvHD - cellules apoptotiques associées à un infiltrat inflammatoire sont recherchés sur la langue qui présente constamment des lésions [21]. Le degré d'atteinte est évaluée selon la classification de Fujiwara [22]. Le grade 1 correspond à un envahissement du chorion par un infiltrat lymphocytaire focal. Dans le grade 2, l'infiltrat est associé à des cellules basales apoptotiques. Dans le grade 3, la lame basale est touchée, l'atteinte des cellules épithéliales est diffuse et l'infiltrat envahit l'épithélium, qui conserve encore son intégrité. Dans le grade 4, il y a de nombreuses cellules apoptotiques avec de larges plages de décollement de l'épithélium et un infiltrat diffus dans le chorion et très dense dans l'épithélium.

Dans les autres organes cibles, à savoir le foie, la peau et le tube digestif, on observe également les lésions histologiques caractéristiques de la GvHD. L'apoptose est confirmée par marquage immunohistochimique des brins d'ADN fragmentés des cellules apoptotiques (technique TUNEL), selon la technique développée par Gavrieli [23]. Les coupes déparaffinées sont incubées dans $20 \mu \mathrm{g} / \mathrm{ml}$ de protéinase $\mathrm{K}$ (Boehringer Mannheim,
Indianapolis, USA), à température ambiante, pendant 30 minutes. Après blocage de l'action de la péroxydase endogène par le péroxyde d'hydrogène à $2 \%$ (Sigma, Saint Louis, USA), les tissus sont incubés pendant 12 minutes à $30^{\circ} \mathrm{C}$ dans un mélange de terminal deoxynucleotidyl transferase (TdT) (Roche, France), digoxigenin-labeled UTP and dATP (Roche, France), puis dans une solution d'anticorps anti-digoxigénine biotinylé marqué par la péroxydase. Une contre-coloration est réalisée à l'hématoxyline et au Bluing Reagent (Ventana, France).

\section{RÉSULTATS}

\section{Induction d'une GvHD}

Après injection de 2,5 $10^{7}$ splénocytes totaux allogéniques normaux (wt), l'atteinte clinique des souris receveuses est objectivée dès $\mathrm{J} 3$, par une perte de poids progressive. Celle-ci est de 14,9\% (SD : 6,97) en moyenne à J14 (Fig. 1) et de $21,61 \%(S D: 9,83)$ en moyenne à J28. L'atteinte clinique de la peau et les modifications de la posture et de l'activité restent modérées (grade 1). Les contrôles syngéniques conservent une courbe de poids stable et ne présentent aucune modification clinique.

L'atteinte buccale a été appréciée sur la langue qui présente cliniquement une atteinte constante de ses bords latéraux [21]. Après l'injection de $2,5.10^{7}$ splénocytes, on observe peu d'atteintes histologiques à J3: seules quelques cellules mononucléées sont présentes dans le chorion (Fig. 2A). A partir de J7, un infiltrat de cellules mononucléées (monocytes, macrophages et lymphocytes) se forme dans le chorion et quelques cellules épithéliales basales sont apoptotiques. L'atteinte est de grade 1 selon la classification de Fujiwara. L'infiltrat, localisé initialement dans le chorion, envahi progressivement l'épithélium. Parallèlement à l'accroissement de sa densité, le nombre de cellules épithéliales apoptotiques augmente, pour atteindre son maximum à J14 (Fig. 2 A, 2 B et 2 C). L'atteinte est de grade 3, mais on observe déjà quelques décollements limités de l'épithélium. A J28, l'épithélium est atrophique avec présence de quelques zones présentant des cellules épithéliales apoptotiques. La densité de l'infiltrat est plus faible qu'à J14. 


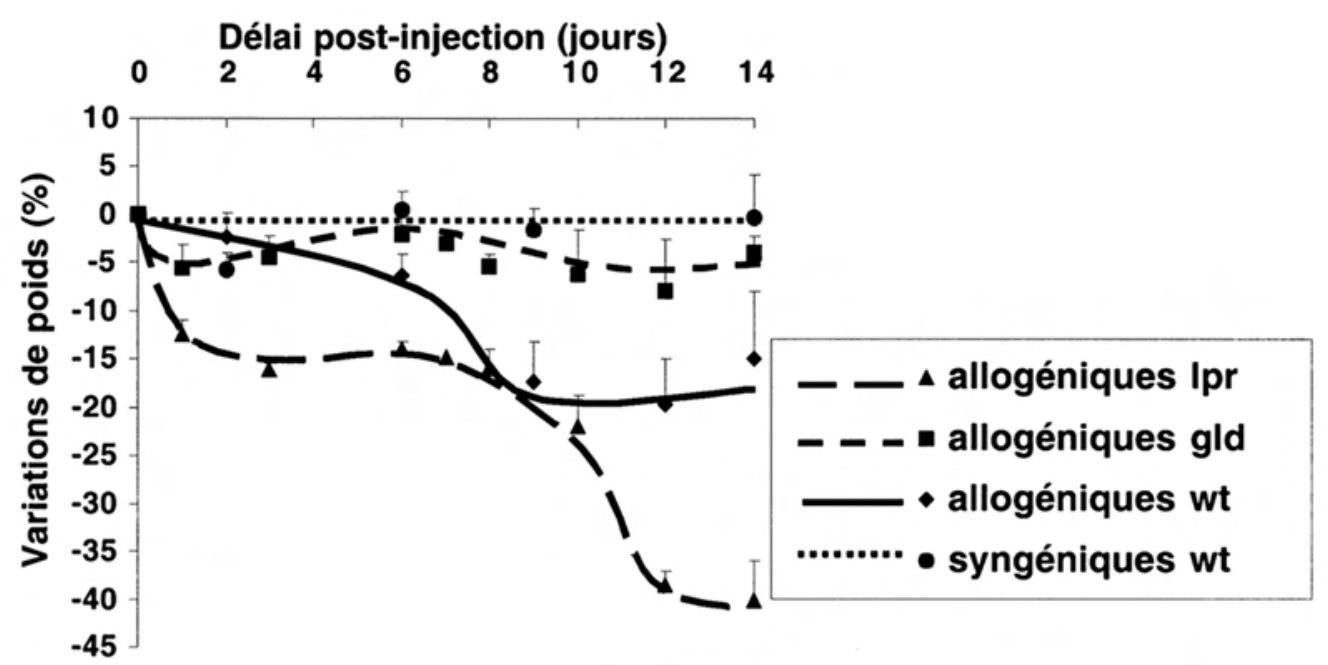

Figure 1 : Courbe de poids (\%) des souris receveuses après injection de $2,5.10^{7}$ splénocytes allogéniques normaux (wt), déficients en CD95L (gld) ou déficients en CD95 (Ipr) ou après injection de 2,5.107 syngéniques wt. Weight loss (\%) of recipients after transfusion of $2.5 \times 10^{7}$ wild type (wt), CD95L deficient (gld) or CD95 deficient (lpr) allogeneic splenocytes or after transfusion of $2.5 \times 10^{7}$ wild type syngeneic splenocytes.

Les contrôles syngéniques ne présentent aucune lésion histologique.

Pour évaluer le rôle des réactions non spécifiques dues aux cellules B et aux cellules NK dans la pathogénèse des lésions de ce modèle, ces cellules ont été éliminées de la suspension de splénocytes et $10^{7}$ lymphocytes $T$ allogéniques purifiés ont été injectés aux souris receveuses.

A J14, l'infiltrat de cellules mononucléées est moins dense que celui observé après injection de $2,5.10^{7}$ splénocytes allogéniques, mais la densité des cellules apoptotiques dans l'épithélium est identique (grade 3). Le transfert de lymphocytes T syngéniques ne s'accompagne pas de perte de poids, ni de lésions histologiques.

\section{Sévérité des lésions en fonction du nombre de splénocytes injectés}

Pour apprécier si l'intensité des lésions est proportionnelle au nombre de cellules allogéniques injectées, différentes quantités de splénocytes allogéniques normaux ont été injectés chez des receveurs immunodéficients. Quatorze jours après injection de $10^{6}$ splénocytes totaux, on observe une perte de poids de $7,71 \%$ (SD : 3,6) en moyenne. On note en microscopie optique, à $\mathrm{J} 14$, une GvHD modérée de grade 1, selon la classification de Fujiwara. Le nombre de cellules mononucléées dans le derme est faible et il n'y a que quelques cellules épithéliales basales apoptotiques. Par contre, l'injection de $10^{7}$ splénocytes totaux provoque une perte de poids de $9,97 \%$ (SD : 2,9) en moyenne, un infiltrat intradermique plus dense et une apoptose plus marquée des cellules épithéliales. L'atteinte est de grade 2 .

L'injection de $5.10^{7}$ splénocytes totaux provoque une GvHD importante, multiviscérale, accompagnée d'une altération de l'état général des receveurs et une perte de poids de 12,44\% (SD : 5,2) en moyenne à J14. La muqueuse linguale présente un infiltrat inflammatoire important avec des décollements de l'épithélium et des foyers de nécrose et d'apoptose. L'atteinte est de grade 3 , voire 4.

L'injection de 108 splénocytes totaux provoque la mort des souris à $\mathrm{J} 2$, la perte de poids moyenne est peu importante (5\%; SD : 2,3), mais l'atteinte clinique est sévère (grade 4). L'infiltrat de cellules mononucléées est peu dense, mais on observe de nombreuses cellules apoptotiques, accompagnées de larges plages de décollement de l'épithélium. L'injection d'un nombre identique de cellules syngéniques ne s'accompagne pas d'altération de l'état général, ni de lésions histologiques. médecine

buccale chirurgie buccale

VOL. $9, N^{\circ} 3$ 2003

page 153 

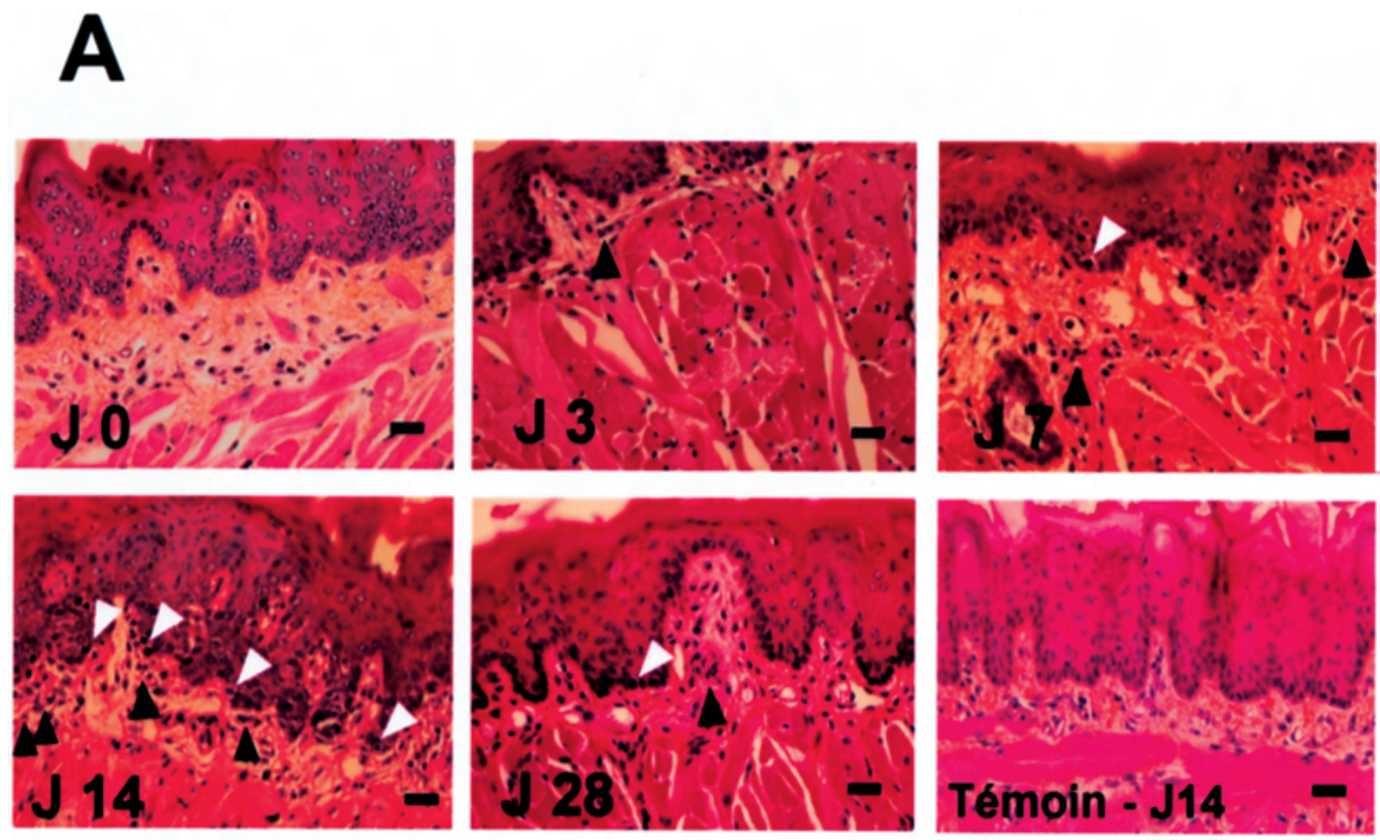

médecine

buccale

chirurgie

buccale

VOL. $9, \mathrm{~N}^{\circ} 3$ 2003

page 154
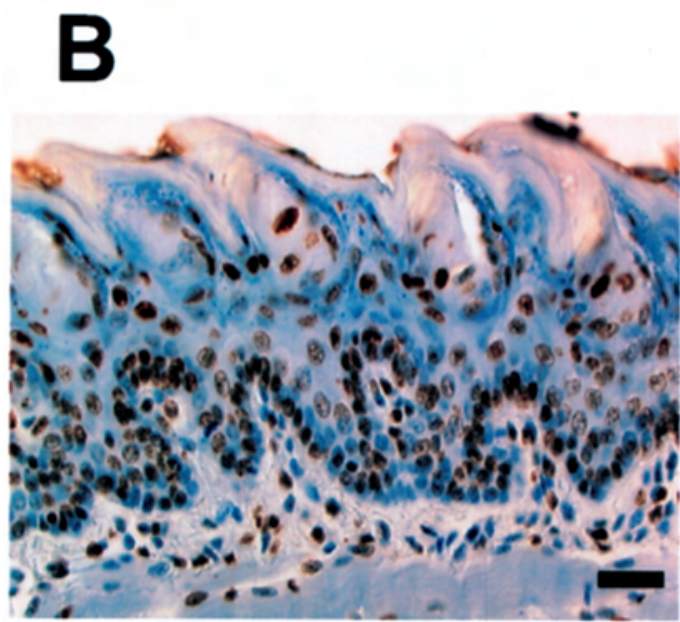
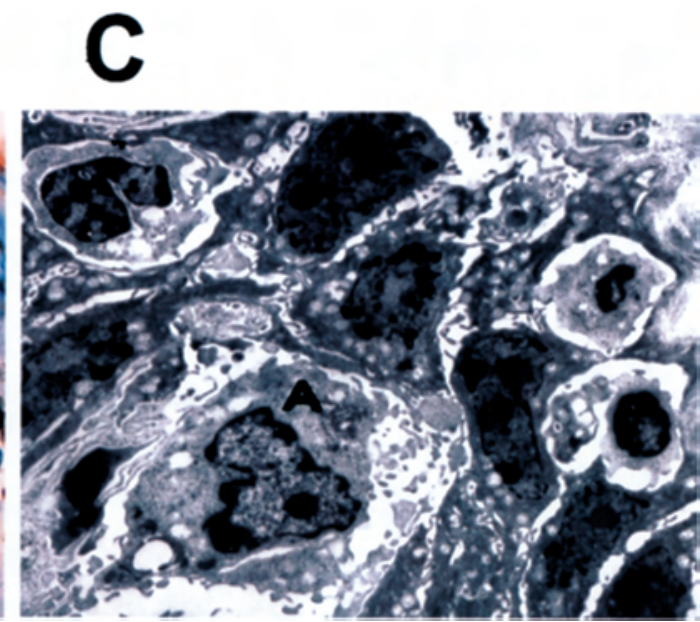

Figure 2 : A : biopsies de la muqueuse linguale à JO, J3, J7, J14 et J28 après l'injection de 2,5.107 splénocytes allogéniques normaux et à J14 après l'injection de 2,5 107 splénocytes syngéniques normaux (Témoin J14) - coloration hématoxyline-éosine $(\mathrm{HE})$, échelle $=50 \mu \mathrm{m}$ : les cellules apoptotiques sont indiquées par des flèches blanches et l'infiltrat mononucléé par des flèches noires.

$\mathrm{B}$ : coloration TUNEL à J14 après l'injection de $2510^{7}$ splénocytes allogéniques normaux : les cellules colorées en brun correspondent à des cellules apoptotiques et les cellules bleues sont non apoptotiques - échelle $=50 \mu m$ $\mathrm{C}$ : microscopie électronique d'une biopsie à $\mathrm{J} 14$ après l'injection de $2510^{7}$ splénocytes allogéniques normaux - $\mathrm{X}$ 16000 : « $A$ » indique un kératinocyte apoptotique

A : Biopsies of tongue mucosa 0, 3,7, 14 and 28 days after transfusion of $2.5 \times 10^{7}$ allogeneic wild type splenocytes and after transfusion of $2.5 \times 107$ syngeneic wild type splenocytes at day 14 (Témoin J14) - Hematin Eosin coloration, bar scale $=50 \mu \mathrm{m}:$ white arrows indicate apoptotic cells and black arrows indicate infiltrate.

$B$ : TUNEL assay 14 days after transfusion of $2.5 \times 10^{7}$ allogeneic wild type splenocytes : apoptotic cells are brown, when non apoptotic cells are blue - bar scale $=50 \mu \mathrm{m}$

$C$ : Electron microscopy of a biopsy 14 days after transfusion of $2.5 \times 10^{7}$ allogeneic wild type splenocytes $-x 16,000$ : « $A$ » indicate apoptotic keratinocyte 
Wt

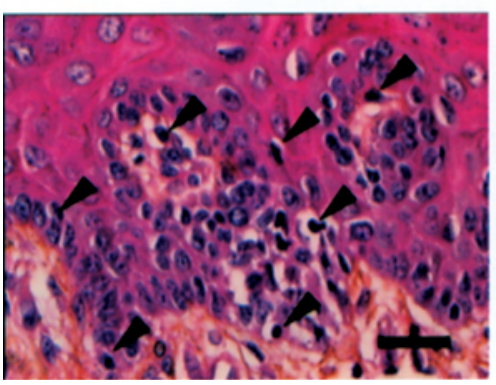

Gld

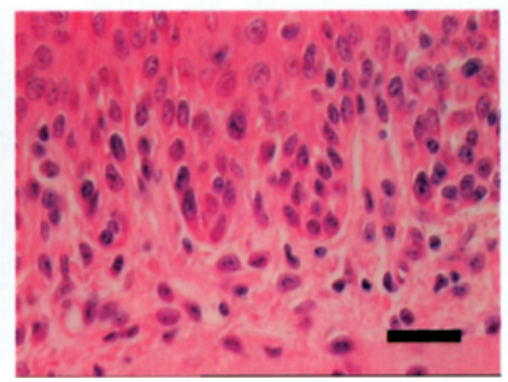

Lpr

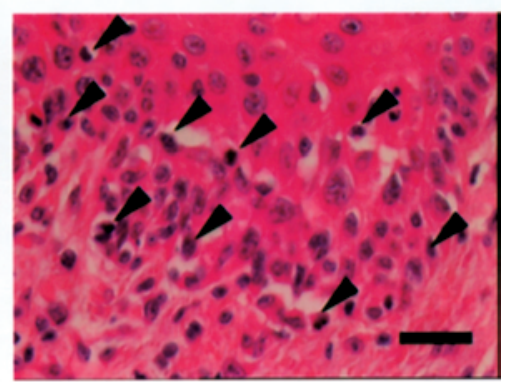

Figure 3 : Biopsies de la muqueuse linguale à $\mathrm{J} 14$ après le transfert de 2,5 $10^{7}$ splénocytes allogéniques normaux (wt), déficients en CD95L (gld) ou déficients en CD95 (Ipr) : les flèches indiquent les cellules épithéliales apoptotiques - Coloration HE, échelle $=50 \mu \mathrm{m}$

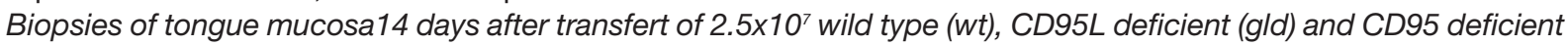
(Ipr) allogeneic splenocytes . Arrows indicate apoptotic epithelial cells - HE coloration, bare scale $=50 \mu \mathrm{m}$

Ainsi, l'atteinte clinique et histologique des receveurs allogéniques dépend du nombre de splénocytes et du nombre de lymphocytes $T$ allogéniques transférés.

\section{Rôle du ligand de CD95 dans la patho- génèse des lésions lichénoïdes}

Pour évaluer, dans la pathogénèse du processus apoptotique des cellules cibles épithéliales, le rôle du ligand de CD95 exprimé par les lymphocytes du donneur, des splénocytes allogéniques déficients en CD95L ont été injectés. Pour vérifier que la mutation du CD95L ne provoque pas d'autres anomalies des cellules $\mathrm{T}$, des contrôles ont été réalisés par injection du même nombre de splénocytes déficients en CD95.

Les souris ayant reçu $2,5.10^{7}$ splénocytes déficients en CD95L fonctionnel (g/d/C57BL/6) présentent une atteinte clinique minime et une perte de poids de 4,02\% (SD : 1,82) en moyenne à J14 (Fig. 1). Par contre, les souris ayant reçu 2,5.107 splénocytes déficients en CD95 (Ipr/C57BL/6) présentent une perte de poids de 39,3\% (SD: 4) en moyenne et une atteinte clinique de grade 4. A J14, l'injection de cellules T déficients en CD95L n'entraîne pas l'apparition de lésion apoptotique malgré la présence d'un infiltrat de cellules mononucléées dans le chorion (Fig. 3). Par contre, l'injection de cellules T dépourvues en CD95 induit le développement de lésions linguales très importantes avec plus de cellules épithéliales apoptotiques et un infiltrat plus dense que lors de l'injection de $2,5.10^{7}$ splénocytes normaux ; l'atteinte est de grade 3 .

Les contrôles réalisés par injection de cellules syngéniques normales, déficientes en CD95 ou en ligand de CD95 fonctionnel, avec des souris immunodéficientes RAG-C57BL/6, ne présentent pas de lésions.

\section{DISCUSSION}

Les organes cibles de la GvHD chez les malades ayant reçu une greffe de moelle osseuse sont le foie, le tube digestif et la peau. La muqueuse de la cavité buccale constitue également une cible précoce dans la GvHD [16, 21] et la lésion élémentaire est de nature lichénoïde. Elle est caractérisée par une apoptose des cellules cibles au voisinage de cellules mononucléées. Dans le modèle murin utilisé, son expression est maximale à $\mathrm{J} 14$ et elle est présente dans tous les organes cibles caractéristiques de la GvHD [15]. Sur le plan histologique, l'apoptose de la cellule se déroule en 3 phases. Tout d'abord, la chromatine se condense et se rapproche de la membrane nucléaire. La cellule diminue en volume tout en conservant l'intégrité de ses organites intracytoplasmiques. Puis, l'activation d'endonucléases non lysosomiales permet de couper le double brin d'ADN entre les nucléosomes, morcelant ainsi la chromatine en fragments d'environ 185 paires de bases, voire en fragments plus gros du fait du regroupement de celle-ci. Le noyau se médecine

buccale chirurgie buccale

VOL. $9, N^{\circ} 3$ 2003

page 155 
médecine

buccale

chirurgie

buccale

VOL. $9, \mathrm{~N}^{\circ} 3$ 2003

page 156 fragmente en plusieurs morceaux et la cellule émet alors des prolongements contenant des fragments nucléaires. Enfin, la cellule, exprimant des facteurs d'endocytose tel la phosphatidine sérine, est phagocytée rapidement par les macrophages ${ }^{[24]}$ ou persiste in situ sous forme de corps apoptotiques.

La localisation préférentielle des cellules apoptotiques, ou corps colloïdes, dans l'épithélium, est la couche basale. L'augmentation du nombre des lésions cellulaires provoque alors la rupture de la membrane basale et induit la formation de bulles dans les cas sévères.

L'infiltration de cellules mononucléées est également un des critères pour le diagnostic de la GvHD [8, 25]. Dans le modèle utilisé, l'infiltrat envahit progressivement le chorion avant l'apparition des lésions apoptotiques épithéliales et l'augmentation de sa densité s'accompagne d'une augmentation du nombre de lésions apoptotiques dites « satellit cell necrosis ", ainsi que l'a montré Deeg [26]. L'envahissement de l'épithélium par les cellules mononuclées s'accompagne d'une augmentation du nombre de cellules apoptotiques, comme dans les lésions du lichen plan [27, 28]. Dans ce modèle, l'atteinte lichénoïde du revêtement muqueux buccal ressemble donc à celle du lichen plan idiopathique [29, 30], qui associe une apoptose des cellules basales de l'épithélium et un infiltrat lymphocytaire sous- épithélial avec exocytose. Cette ressemblance suggère alors un ou plusieurs mécanisme(s) effecteur(s) commun(s) pour ces pathologies.

Plusieurs types cellulaires sont impliqués dans la pathogénèse de ces lésions, à savoir les populations lymphocytaires T CD4+ et CD8+ [31, 32], les cellules B [33], les cellules NK [27] et les macrophages [34, 35]. Dans le modèle utilisé, les lésions induites par les cellules T purifiées sont identiques à celles induites par une suspension cellulaire contenant des cellules T, B et NK. II semble donc que les cellules $T$ soient les principaux effecteurs dans les lésions lichénoïdes [36]. De plus, la sévérité du processus apoptotique est proportionnelle au nombre de cellules $T$ effectrices injectées, donc à la densité de l'infiltrat en lymphocytes $T$ allogéniques présent à proximité des cellules cibles [26]. La sévérité de l'atteinte est également accrue lorsque les cellules $T$ de l'infiltrat sont déficientes en CD95 (Ipr-C57BL/6), parce que, étant moins sensibles à l'apoptose médiée par CD95/CD95L [37], elles ont un meilleur potentiel de survie [38].

Une étude récente a montré que l'apoptose des cellules endothéliales est médiée par l'expression de CD95L par les lymphocytes T effecteurs de la réaction allogénique [15]. L'injection de splénocytes totaux ou de cellules $T$ allogéniques provenant de souris déficientes en protéine CD95L (cellules gld-C57BL/6) ne provoque pas de lésion apoptotique au niveau des cellules épithéliales de la muqueuse buccale. II semblerait donc que l'induction de l'apoptose au niveau des cellules épithéliales buccales observée dans la GvHD et dans le lichen idiopathique soit également lié à l'expression de CD95L par les cellules effectrices issues du donneur.

Dans le même travail sur la cellule endothéliale [15], il a été montré que le blocage de l'activation de la caspase-8, la première caspase activée par la ligation de CD95 [39], provoque une interruption du signal de mort cellulaire et donc une prévention de l'apoptose épithéliale. Ainsi, une des voies de prévention des lésions épithéliales de la GvHD, mais également des lésions du lichen plan idiopathique, pourrait être réalisée par blocage du signal apoptotique intracellulaire initié par la ligation du ligand de CD95 au récepteur membranaire CD95 exprimé par des cellules cibles.

Le modèle murin étudié développe une réaction allogénique systémique disséminée qui mime les lésions typiques de la GvHD. L'atteinte buccale lichénoïde se traduit par une lésion élémentaire précoce et reproductible, similaire à celle du lichen plan idiopathique. Celle-ci associe une apoptose des cellules basales épithéliales et un infiltrat mononucléé satellite effecteur.

Ce modèle murin a permis de montrer que l'apoptose des cellules cible est proportionnelle à la quantité de lymphocytes $T$ effecteurs recrutés progressivement et s'accumulant dans le chorion. L'expression de CD95L par les lymphocytes T activés de l'infiltrat serait responsable de l'apoptose des cibles épithéliales satellites. 


\section{RÉFÉRENCES}

1 - Salonen L, Axell T, Hellden L. Occurrence of oral mucosal lesions, the influence of tobacco habits and an estimate of treatment time in an adult swedish population. J Oral Pathol Med 1990; 1: 170-6.

2 - Murti PR, Daftary DK, Bhonsle R, Gupta PC, Mehta FS, PINBORG JJ. Malignant potential of lichen planus: observation in 722 patients from India. J Oral Pathol 1986; 15: 71-7.

3 - Holmstrup P, ThORM JJ, Rindum J, PInBORG JJ. Malignant development of oral lichen planus affected oral mucosa. J Oral Pathol 1988; 17: 219-25.

4 - Sugerman PB, Savage NW, Walsh LJ, Seymour GJ. Disease mechanisms in oral lichen planus. A possible role for autoimmunity. Aust J Dermatol 1993; 34: 63-9.

5 - Simon JR, Reimer G, Schardt M, HoRnstein OP. Lymphotoxicity for oral mucosa in lichen planus. Dermatologica 1983; 167: 11-5.

6 - Sugerman PB, Rollason PA, Savage NW, Seymour GJ. Suppressor cell function in oral lichen planus. J Dent Res 1992; 71: 1916-9.

7 - Walsh LJ, Savage NW, Ishil T, Seymour GJ. Immunopathogenesis of oral lichen planus. J Oral Pathol Med 1990; 19: 389-96.

8 - SNOVER DC. The pathology of acute graft versus host disease (pp. 337-53). In: Graft-vs host disease. Immunology, pathophysiology and treatment. J. Ferrara, H.J. Deeg, S.J. Burakoff. éds: Marcel Dekker, New York 1990.

9 - Janin-Mercier A, Saurat JH, Bourges M, Sohier J, JEAN LD, GLuCKMAN E. The lichen planus like and sclerotic phases of the graft versus host disease in man: an ultrastructural study of six cases. Acta Derm Venereol 1981; 61: 187-93.

10 - LoWIn B, PeITsch MC, Tschopp J. Perforin and granzymes: crucial effector molecules in cytolytic T lymphocyte and natural killer cell-mediated cytotoxicity. Curr Top Microbiol Immunol 1995; 198: 1-24.

11 - Blazar BR, TAYlor PA, Vallera DA. CD4+ and CD8+ T cells each can utilize a perforin-dependent pathway to mediate lethal graft-versus-host disease in major histocompatibility complex-disparate recipients. Transplantation 1997; 64: 571-6.

12 - Piguet PF, Grau Ge, Allet B, Vassalli P. Tumor necrosis factor/cachectin is an effector of skin and gut lesions of the acute phase of graft-vs.-host disease. J Exp Med 1987; 166: 1280-9.

13 - HiROYasu S, Shiraishi M, KoJI T, Mamadi T, SugaWa H, TOMORI H, Muto Y. Analysis of Fas system in pulmonary injury of graft-versus-host disease after rat intestinal transplantation. Transplantation 1999; 68: 933-8.

14 - Murai M, Yoneyama H, Harada A, YI Z, VestergaArd C, Guo B, SUZUKI K, AsakURA H, Matsushima K. Active participation of CCR5(+)CD8(+) T lymphocytes in the pathogenesis of liver injury in graft-versus-host disease. J Clin Invest 1999; 104: 49-57.
15 - Janin A, Deschaumes C, Daneshrouy M, Estaquier J, Micic-Polianski J, Rajagopalan-LeVasseur P, AKarid K, Mounier N, Gluckman E, Socie G, Ameisen JC. CD95 engagement induces disseminated endothelial cell apoptosis in vivo: immunopathologic implications. Blood 2002; 99: 2940-7.

16 - Sale GE, Lerner KG, Barker EA, Shulman HM, THOMAS ED. The skin biopsy in the diagnosis of acute graft-versus-host disease in man. Am J Pathol 1977; 89: 621-35.

17 - MURAKI Y, YOSHIOKA C, FUKUDA J, HANEJI T, KOBAYASHI N. Immunohistochemical detection of Fas antigen in oral epithelia. J Oral Pathol Med 1997; 26: 57-62.

18 - NAGATA S, SUDA T. Fas and Fas ligand: Ipr and gld mutations. Immunol Today 1995; 16: 39-43.

13 - TAKAHASHI T, TANAKA M, BranNan Cl, JENKINS NA, COPELAND NG, SUdA T, NAGATA S. Generalized lymphoproliferative disease in mice, caused by a point mutation in the Fas ligand. Cell 1994; 76: 969-76.

20 - CoOke KR, Kobzik L, Martin TR, Brewer J, Delmonte J, JR., Crawford JM, Ferrara JL. An experimental model of idiopathic pneumonia syndrome after bone marrow transplantation: I. The roles of minor $\mathrm{H}$ antigens and endotoxin. Blood 1996; 88: 3230-9.

21 - SAlE GE, RafF RF, Storb R. Stem cell regions in filiform papillae of tongue as targets of graft-versus-host disease. Transplantation 1994; 58: 1273-5.

22 - FuJIWARA K, SAKAI Y, SugIURA J, YAMASAKI A. Oral mucosal lesions in experimental graft-versus-host disease: morphological and immunohistochemical characterization of infiltrating cells. J Oral Pathol Med 1996; 25: 314-9.

23 - GAVRIELI Y, SheRman Y, BEn-SASSON SA. Identification of programmed cell death in situ via specific labeling of nuclear DNA fragmentation. J Cell Biol 1992; 119: 493501.

24 - Ferrara J, Guillen FJ, Sleckman B, Burakoff SJ, MURPHY GF. Cutaneous acute graft-versus-host disease to minor histocompatibility antigens in a murine model: histologic analysis and correlation to clinical disease. J Invest Dermatol 1986; 86: 371-5.

25 - HORN TD, ZAHURAK ML, ATKINS D, SOLOMON AR, VoGELSANG GB. Lichen planus-like histopathologic characteristics in the cutaneous graft-vs-host reaction. Prognostic significance independent of time course after allogeneic bone marrow transplantation. Arch Dermatol 1997; 133: 961-5.

26 - DEEG HJ. Graft-versus-host disease and the development of late complications. Transfus Sci 1994; 15: 24354.

27 - Thomas DW, Matthews JB, Prime SS. Mucosal cellmediated immunological changes associated with experimental graft-versus-host disease. J Oral Pathol Med 1996; 25: 145-50.

\begin{tabular}{l}
$\begin{array}{l}\text { médecine } \\
\text { buccale } \\
\text { chirurgie } \\
\text { buccale }\end{array}$ \\
\hline VOL. $9, N^{\circ} 3$ \\
2003 \\
\hline page 157
\end{tabular}


28 - Bloor BK, Malik FK, Odell EW, Morgan PR. Quantitative assessment of apoptosis in oral lichen planus. Oral Surg Oral Med Oral Pathol Oral Radiol Endod 1999; 88: 187-95.

29 - BARRETT AP, BiLous AM. Oral patterns of acute and chronic graft-v-host disease. Arch Dermatol 1984; 120 : 1461-5.

30 - Schubert MM, Sullivan KM. Recognition, incidence, and management of oral graft-versus-host disease. $\mathrm{NCl}$ Monogr 1990: 135-43.

31 - Blazar BR, TAYLor PA, Gray GS, Vallera DA. The role of $T$ cell subsets in regulating the in vivo efficacy of CTLA4-Ig in preventing graft-versus-host disease in recipients of fully $\mathrm{MHC}$ or multiple minor histocompatibility-disparate donor inocula. Transplantation 1994; 58: 1422-6.

32 - Blazar BR, Sharpe AH, Taylor PA, PanoskaltsisMortari A, Gray GS, Korngold R, Vallera DA. Infusion of anti-B7.1 (CD80) and anti-B7.2 (CD86) monoclonal antibodies inhibits murine graft-versus-host disease lethality in part via direct effects on CD4+ and CD8+ T cells. J Immunol 1996; 157: 3250-9.

33 - Matsue K, Lum LG, Witherspoon RP, Storb R. Proliferative and differentiative responses of $\mathrm{B}$ cells from human marrow graft recipients to $T$ cell-derived factors. Blood 1987; 69: 308-15.
34 - Nestel FP, Price KS, Seemayer tA, Lapp WS. Macrophage priming and lipopolysaccharide-triggered release of tumor necrosis factor alpha during graft-versus-host disease. J Exp Med 1992; 175: 405-13.

35 - SIMON M, JR., HUNYADI J, GOMEZ RS. Macrophage markers 25F9 and 27E10 on human keratinocytes in normal and diseased skin. J Dermatol 1993; 20: 618-22.

36 - FUJII H, OHASHI M, NAGURA H. Immunohistochemical analysis of oral lichen-planus-like eruption in graft-versus-host disease after allogeneic bone marrow transplantation. Am J Clin Pathol 1988; 89: 177-86.

37 - COHEN PL, EISENBERG RA. The lpr and gld genes in systemic autoimmunity: life and death in the Fas lane. Immunol Today 1992; 13: 427-8.

38 - IWAI H, TOMOdA K, HOSAKa N, MiYASHIMA S, SUSUKa Y, IDEKA H, LEE S, INABA M, IKEHARA S, YamaShITA T. Induction of immune mediated hearing loss in scid mice by injection of MRL/Ipr mouse spleen cells. Hear Res 1998; 117: 173-7.

39 - NAGATA S. Apoptosis by death factor. Cell 1997; 88: 355-65. buccale

chirurgie

buccale

VOL. $9, \mathrm{~N}^{\circ} 3$ 2003 